\title{
REMARQUE \\ SUR UNE RELATION DENDROMÉTRIQUE INATTENDUE CONSÉQUENCES MÉTHODOLOGIQUES \\ POUR LA CONSTRUCTION DES TABLES DE PRODUCTION
}

\author{
N. DECOURT \\ Station de sylviculture et de Production \\ Centre national de Recherches forestières, I.N.R.A., \\ Champenoux - 54370 Einville
}

\section{RÉSUMÉ}

La relation étudiée relie, pour des peuplements équiennes, de même espèce et dans une région donnée, la circonférence moyenne $\mathrm{Cg}$, la hauteur moyenne $\mathrm{Hg}$ et le nombre de tiges sur pied. Cette relation découle de la nature des peuplements étudiés et des traitements subis par ceux-ci. Pour l'utiliser valablement dans la construction de tables de production, on doit tenir compte de ces conditions au moment du choix du matériel et du modèle retenu pour la table.

\section{I. - POSITION DU PROBLÈME}

Lors de la construction des premières tables de production françaises, j'utilise parmi les relations caractérisant la structure d'un ensemble de peuplements d'une même essence et d'une même région, équiennes et purs, mais d'âge, de densité et de classes de productivité parfois très variés, une équation reliant la circonférence moyenne $\mathrm{Cg}$ au nombre de tiges sur pied $\mathrm{N}$ et à la hauteur moyenne du peuplement $\mathrm{Hg}$ :

$$
\mathrm{Cg}=f\left(\mathrm{~N}, \mathrm{H}_{\mathrm{g}}\right)
$$

Cette équation, établie en 1965 pour le Pin Sylvestre et pour le Pin Laricio de Corse en Sologne (Decourt, 1965) avait la forme suivante :

$a_{1}, b_{1}, c_{1}$ étant des constantes.

$$
\mathrm{Cg}=a_{1}+b_{1} \cdot \frac{10^{4}}{\mathrm{~N}}+c_{1} \cdot \mathrm{H}_{\mathrm{g}}
$$


L'ajustement était excellent avec plus de 97 p. 100 de la variation des Cg «expliquée» par l'équation et des écarts-types de l'ordre de $3-4 \mathrm{~cm}$ sur la circonférence. L'introduction de l'âge des peuplements dans l'équation ne l'améliorait pas de façon significative.

Par la suite, d'autres équations du même type ont été utilisées pour d'autres espèces : le Douglas, l'Épicéa commun, le Pin maritime dans différentes régions de France (DECOURT, 1967, 1969, 1972, 1973). L'ajustement s'est toujours révélé excellent, l'âge n'intervenant pas, ou rarement et de façon très peu significative. Parmi les formes d'équations utilisées avec succès, citons également :

$$
\begin{aligned}
\text { Log. } \mathrm{C}_{\mathrm{g}} & =a_{2}+b_{2} \cdot \log . \mathrm{N}+c_{2} . \text { Log. } \mathrm{H} \\
\mathrm{C}_{\mathrm{g}} & =a_{3}+b_{3} \cdot \mathrm{H}_{\mathrm{g}}+c_{3} \cdot \mathrm{H}_{\mathrm{g}}{ }^{2} \cdot \mathrm{N}
\end{aligned}
$$

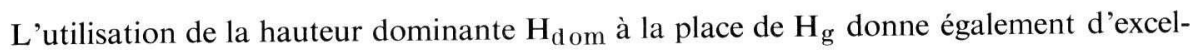
lents résultats, quoique parfois légèrement moins bons.

S. O. ANDERson (1963) utilise également une liaison entre diamètre moyen, hauteur dominante et nombre de tige, mais uniquement pour le calcul des valeurs initiales, avant éclaircie, de ses tables de production pour le Pin Sylvestre.

La relation (1) a depuis été utilisée sous des formes explicites diverses par différents chercheurs, soit pour construire des tables de production (A. Pita Carpenter et A. MadriGAL, 1973) soit pour définir des normes d'éclaircie (AвETZ, 1973).

Ces résultats sont à rapprocher de l'équation proposée par F. EvERT (1971) qui relie linéairement le diamètre quadratique moyen $\mathrm{D}_{\mathrm{g}}$ à une variable indépendante, combinant la hauteur moyenne du peuplement (la hauteur de LOREY : H L), le nombre de tiges sur pied et également l'âge $\mathrm{T}$ du peuplement. Cette équation a la forme suivante :

$$
\mathrm{D}_{\mathrm{g}}=a_{4}+b_{4} \cdot \sqrt[4]{\frac{\mathrm{H} \mathrm{L} \cdot \mathrm{T}}{\mathrm{N}}}
$$

L'ajustement de cette équation à partir de nos données s'est révélé excellent, mais pas supérieur à ceux obtenus à partir d'équations du type (2) ou (3) qui ne font pas intervenir T, autrement que par l'intermédiaire de $\mathrm{H}_{\mathrm{g}}$.

Finalement, notre équation $\mathrm{C}_{\mathrm{g}}=f\left(\mathrm{H}_{\mathrm{g}}, \mathrm{N}\right)$ semble d'une façon générale bien vérifiée. Une liaison étroite de ce type est pourtant inattendue pour un ensemble de peuplements souvent très différents par l'âge, la classe de productivité et soumis à des éclaircies variées.

En fait, ce résultat correspond à un certain nombre d'hypothèses très souvent vérifiées dans les peuplements utilisés pour établir cette relation.

Dans la mesure où on veut l'utiliser, il importe de faire une analyse serrée de ces hypothèses. Elles entraînent en effet des contraintes particulières pour le choix des placetteséchantillons, mais également pour l'emploi correct de la relation dans la construction de tables de production et notamment de tables de production à régime d'éclaircie variable.

\section{II. - SIGNIFICATION DE L'ÉQUATION $\mathrm{C}_{\mathrm{g}}=f\left(\mathrm{~N}, \mathrm{H}_{\mathrm{g}}\right)$}

Sous le terme « hypothèse », nous désignerons ici soit des lois dendrométriques plus ou moins approchées, mais généralement admises, soit des conditions particulières imposées aux peuplements servant de matériel d'étude. De plus, dans ce qui suit, il ne sera question que de peuplements purs et équiennes, d'une même "région de croissance » (Wuchsgebiet). 
Considérons les surfaces terrières des peuplements. Soit $G$ la surface terrière constatée pour $\mathrm{N}$ tiges sur pied et $\mathrm{G}_{\text {tot }}$ la surface terrière totale définie par la relation :

$$
\mathrm{G}_{\text {тOT }}=\mathrm{G}+\mathrm{GE}
$$

où $\mathrm{G}$ E est la somme des surfaces terrières enlevées en éclaircie ou disparues par mortalité naturelle.

On posera les hypothèses suivantes d'où découle la relation (1)

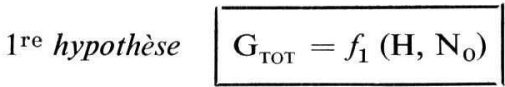

La surface terrière totale " produite » par un peuplement est pour une essence donnée et une région donnée fonction de sa hauteur seule, et dans une moindre mesure de sa densité initiale, caractérisée par le nombre de tiges initiales $\mathrm{N}_{0}$.

Avant de commenter cette proposition, on remarquera qu'il existe le plus souvent une relation permettant d'évaluer le volume d'un peuplement $\mathrm{V}$ ou d'un lot d'arbres par une relation de la forme :

$$
\mathrm{V}=f_{2}(\mathrm{H}, \mathrm{G}) \text {. }
$$

Cette équation, qui permet d'établir de bons tarifs de peuplements (Assmann, 1961; KrenN, 1946; Decourt, 1971; Bouchon, 1973) permet également d'exprimer notre première hypothèse sous la forme suivante :

$f_{3}$ résulte en effet de $f_{1}$ et de $f_{2}$.

$$
\mathrm{V}_{\text {тот }}=f_{3}\left(\mathrm{H}, \mathrm{N}_{\mathrm{O}}\right)
$$

Notons que dans ces trois équations, $\mathbf{H}$ peut désigner soit la hauteur moyenne $\mathbf{H}_{\mathrm{g}}$, soit la hauteur dominante $\mathrm{H}_{\mathrm{d} o m}$. Ces deux hauteurs étant du reste très généralement liées par une relation linéaire étroite (éventuellement améliorée par la prise en compte du nombre de tige $\mathrm{N}$ comme variable indépendante). Notons également qu'une relation analogue à $f_{2}$ existe probablement aussi pour la biomasse (DECOURT, 1973).

Plusieurs travaux sur les relations entre le rendement et la densité dans les peuplements végétaux (notamment KIRA et al., 1953 et KIRA et SHINOZAKI, 1956) ont établi que pour une densité initiale donnée et sur une station donnée, le rendement total ne dépend que de la densité initiale et de l'âge.

Mieux, avec le temps, l'effet de la densité initiale s'atténue et le rendement final est sensiblement constant pour un éventail très large de densité initiale.

Cette loi a été vérifiée pour les peuplements forestiers (PienaAr L. V. et Turnbull K. J., 1973) et pour leur croissance en surface terrière.

Si on remarque qu'on a observé dans un très grand nombre de cas que la croissance en hauteur ne dépend pas de la densité, la loi de « constance du rendement final » énoncée cidessus n'est autre pour les peuplements forestiers qu'une forme limite de la loi dite de EICHHORN élargie.

Cette loi stipule en effet que la production totale en volume ne dépend que de la hauteur atteinte par le peuplement. Bien vérifiée pour la hauteur dominante, pratiquement indé- 
pendante de la densité, cette loi l'est de façon plus approchée pour la hauteur moyenne, plus dépendante de la densité.

On pourrait donc écrire à la limite :

$$
\begin{aligned}
\mathrm{G}_{\text {тот }} & =f_{1}(\mathrm{H}) \\
\text { et } \mathrm{V}_{\text {тот }} & =f_{3}(\mathrm{H})
\end{aligned}
$$

En fait, admettre l'hypothèse moins forte proposée ci-dessus ne gêne pas la suite du raisonnement.

On peut donc considérer comme bien établi par un ensemble d'expériences et d'interprétations cohérentes une proposition de la forme :

ou encore :

$$
\begin{aligned}
& \mathrm{G}_{\text {тот }}=f_{1}\left(\mathrm{H}_{\mathrm{g}}, \mathrm{N}_{\mathrm{o}}, \mathrm{N}\right) \\
& \mathrm{G}_{\text {тот }}=f_{4}\left(\mathrm{H}_{\mathrm{dom}}, \mathrm{N}_{\mathrm{o}}\right) .
\end{aligned}
$$

$$
\text { 2e hypothèse } \mathrm{G} \mathrm{E}=f_{5}\left(\mathrm{~N}_{\mathrm{o}}-\mathrm{N}, \mathrm{H}\right)
$$

La somme des surfaces terrières enlevées en éclaircie est une fonction de la hauteur moyenne ou dominante) et du nombre total de tiges enlevées.

Pour un même nombre de tiges initial et une même hauteur, l'intensité des éclaircies est effectivement bien caractérisée par le nombre de tiges sur pied. C'est du reste ainsi qu'on la chiffre parfois (DECOURT, 1965).

Cette proposition est à rapprocher de la formule proposée par R. MAGIN (1966) :

$$
\mathrm{V}_{\text {тот }}(t)=\mathrm{V}_{\mathrm{o}}+\sum_{t_{\mathrm{o}}}^{t} \Delta \mathrm{V}(s)+\sum_{t_{\mathrm{o}}}^{t} \mathrm{~K} \cdot \Delta \mathrm{N}(s) \cdot \mathrm{V}_{\mathrm{m}}(s / 2) .
$$

où $\mathrm{V}_{\text {тот }}(t)$ est la production totale à l'âge $t$

$\mathrm{V}_{\mathrm{o}} \quad$ le volume sur pied initial, à l'âge $t_{0}$

$\Delta \mathrm{V}(s)$ les différences de volumes pour des périodes de durée $s$ de l'ordre de 5 à 10 ans.

$\Delta \mathrm{N}(s)$ les différences de nombre de tiges pour ces mêmes périodes.

$v_{\mathrm{m}}(s / 2)$ le volume de la tige moyenne au milieu des périodes considérées.

les signes $\sum_{t_{0}}^{t}$ signifient qu'on fait la somme des quantités concernées pour toutes les périodes de durée $s$ comprises entre $t_{\mathrm{O}}$ et $t$.

$\mathrm{K}$ est un facteur de correction qui serait valable pour toutes les essences, toutes les classes de productivité et tous les régimes d'éclaircie.

En fait, dans la formule, les deux premiers termes de la somme expriment le volume sur pied et le dernier terme la somme des volumes enlevés en éclaircie entre $t_{0}$ et $t$. Le facteur $\mathrm{K}$ représente donc à peu près le rapport de l'arbre moyen enlevé en éclaircie à l'arbre moyen sur pied. K doit donc dépendre du régime d'éclaircie. J. Franz (1966) a du reste établi empiriquement une formule donnant $\mathrm{K}$ en fonction de $\Delta \mathrm{N}$ et du nombre de tiges sur pied. 
En définitive, ainsi modifiée et quoique ne faisant pas intervenir la nature de l'éclaircie (plutôt par le haut ou plutôt par le bas par exemple), la formule se rapproche du modèle utilisé pour construire nos tables de production provisoires à partir de placettes temporaires.

Si de plus, on remarque que $v_{m}(s / 2)$ est le quotient d'un volume sur pied par le nombre de tiges et que les volumes peuvent s'exprimer en fonction des surfaces terrières et des hauteurs (par la relation $f_{2}$ ci-dessus), la formule de MAGIN se rapproche beaucoup de $f_{5}$, puisqu'elle exprime $G_{\text {тот }}$ en fonction de $G$, de $H$, de $N_{0}$, et de la succession des valeurs de $\mathrm{G}$, de $\mathbf{H}$ et de $\mathrm{N}$, ce qui lui permet sans doute de serrer la réalité de plus près.

Notre hypothèse est évidemment plus fragile. Elle est sans doute valable en moyenne pour un ensemble de peuplements ayant subi le même type d'éclaircies, celles-ci étant réparties en gros, de la même façon dans le temps (rotations de 5 à 10 ans par exemple).

$$
\text { 3e hypothèse } \mathrm{N}_{\mathrm{o}}=\text { constante }
$$

Cela suppose que les peuplements considérés ont tous à peu près la même densité initiale. Cette hypothèse est assez généralement vérifiée dans une région donnée pour les peuplements âgés de plus de 20 ans, les plantations à grand espacement étant d'un usage relativement récent. En cas de semis, naturel ou artificiel, il me semble probable qu'en valeur relative, la constance de $\mathrm{N}_{0}$, autour de valeurs assez fortes, est encore mieux vérifiée.

\section{Conséquence des hypothèses}

Ces trois hypothèses résumées par les relations :

$$
\left\{\begin{array}{l}
\mathrm{G}_{\text {тот }}=f_{1}\left(\mathrm{H}, \mathrm{N}_{\mathrm{o}}\right) \\
\mathrm{G} \mathrm{E}=f_{5}\left(\mathrm{~N}_{\mathrm{O}}-\mathrm{N}, \mathrm{H}\right) \\
\mathrm{N}_{\mathrm{o}}=\mathrm{C}^{\mathrm{te}}
\end{array}\right.
$$

entraînent : $\quad \mathrm{G}=\mathrm{G}_{\text {тот }}-\mathrm{G} \mathrm{E}=f_{6}(\mathrm{~N}, \mathrm{H})$

ce qui se traduit également, puisque $G=N \cdot \frac{C_{g}^{2}}{4 \pi}$, par une relation :

$$
\mathrm{C}_{\mathrm{g}}=f_{7}(\mathrm{~N}, \mathrm{H}) \text {. }
$$

Notons que comme $\mathrm{G}$ est proportionnel à $\mathrm{C}_{\mathrm{g}}^{2}$ (par définition), $\mathrm{C}_{\mathrm{g}}$ est automatiquement connu avec une incertitude environ deux fois moindre que celle constatée sur G. C'est du reste ce qu'on obtient en ajustant les relations par la méthode des moindre carrés à partir de données empiriques.

Il résulte de ces remarques que pour utiliser cette relation dans la construction de tables de production, il ne faut pas trop s'éloigner des hypothèses lors de la récolte des données.

On remarquera cependant que la relation $f_{6}$ implique un phénomène de compensation qui peut la rendre assez stable, lorsque la manière de pratiquer les éclaircies varie. 
Supposons deux éclaircies enlevant la même surface terrière, l'une pratiquée très récemment, l'autre il y a longtemps.

$$
\mathrm{G}=\mathrm{G}_{\text {тот }}(\mathrm{H})-\mathrm{G} \mathrm{E}\left(\mathrm{N}_{\mathrm{O}}-\mathrm{N}, \mathrm{H}\right) .
$$

Dans le cas de l'éclaircie récente, $\mathrm{H}$ est plus grand que dans celui de l'éclaircie ancienne. C'est l'inverse pour $\mathrm{N}_{0}-\mathrm{N}$. Si la forme de l'équation est correcte, elle peut rendre compte de ces deux éclaircies, pourtant très différentes. Cette équation peut du reste contenir $\left(N_{0}-N\right)$ sous des formes diverses. Ce peut être par exemple la différence relative $\frac{N_{0}-N}{N}$ qui intervient, c'est-à-dire finalement le rapport $\frac{\mathrm{N}_{\mathrm{o}}}{\mathrm{N}}$. Ces divers modes d'expression ne changent évidemment rien au raisonnement ci-dessus.

\section{III. - CONSÉQUENCES POUR L'EMPLOI DE LA FORMULE DANS LA CONSTRUCTION DE TABLES DE PRODUCTION}

L'analyse ci-dessus entraîne une série de conséquences.

\section{1. - Conséquences sur le choix des placettes.}

Outre les conditions habituelles : peuplements purs, équiennes, « région de croissance » homogène, les placettes doivent être installées dans des peuplements de même densité initiale. Nous avons vu que ces conditions sont à peu près vérifiées pour les tables déjà construites. Pour l'avenir, il faut donc s'efforcer de remplir ces conditions.

\section{2. - Conséquence sur le modèle utilisé.}

La relation $\mathrm{C}_{\mathrm{g}}=f(\mathrm{~N}, \mathrm{H})$ est une conséquence de la loi dite de Eichhorn. (Cf. : hypothèse $\mathrm{n}^{\circ} 1$ ). On doit donc considérer qu'on se trouve dans les conditions où cette loi se trouve vérifiée. Le régime d'éclaircie notamment ne peut correspondre à des intensités assez fortes pour faire baisser la production. Si toutefois les éclaircies pratiquées dans l'ensemble des placettes utilisées abaissent en moyenne la production totale suivant une même loi ne dépendant que de la hauteur, le raisonnement reste valable et s'applique alors à une relation production totale-hauteur constamment inférieure à celle prévue par la relation d'Eichhorn. C'est sans doute ce qui se passe dans le cas des Pins maritimes des Landes traités pour la production de gemme. L'extrapolation à des peuplements nettement plus denses ou à la ligniculture, des tables existantes devraient faire l'objet d'études complémentaires.

D'autre part, en toute rigueur, la décroissance du nombre de tiges sur pied $\mathrm{N}$ doit être exprimée en fonction de la hauteur $\mathrm{H}$ (dominante, ou même moyenne comme nous l'avons vu). En effet, soit $\mathrm{V}=f(\mathrm{~N}, \mathrm{H})$ l'équation tirée de $\mathrm{C}_{\mathrm{g}}=f(\mathrm{~N}, \mathrm{H})$ et d'une équation-tarif 
convenable, l'accroissement total est à tout moment égal à l'accroissement du peuplement sur pied :

$$
\frac{d \mathrm{~V}}{d t}=f_{\mathrm{t}}^{\prime}(\mathrm{N}, \mathrm{H})
$$

Or, cet accroissement est par hypothèse indépendant de N. Cela ne peut être vérifié que si une relation existe entre la décroissance de $\mathrm{N}$, la décroissance de $\mathrm{H}, \mathrm{N}$ et $\mathrm{H}$.

Exemple : $\mathrm{Si} \mathrm{V}=a+b / \mathrm{N}+c \mathrm{H}$

$$
\begin{array}{ll}
\frac{d \mathrm{~V}}{d t}=-\frac{b}{\mathrm{~N}^{2}} \cdot & \frac{d \mathrm{~N}}{d t}+c \frac{d \mathrm{H}}{d t} \\
\frac{d \mathrm{~V}}{d \mathrm{H}}=-\frac{b}{\mathrm{~N}^{2}} \cdot & \frac{d \mathrm{~N}}{d \mathrm{H}}+c
\end{array}
$$

la condition s'écrira :

$$
-b / \mathrm{N}^{2} \cdot \frac{d \mathrm{~N}}{d \mathrm{H}}=g(\mathrm{H})
$$

L'ajustement de la relation $\mathrm{C}_{\mathrm{g}}=f(\mathrm{~N}, \mathrm{H})$ se fait à partir de données telles qu'une relation existe bien entre $d \mathrm{~N}, \mathrm{~d} \mathrm{H}, \mathrm{H}$ et $\mathrm{N}$.

Pour les tables déjà construites, on s'est finalement placé à peu près dans ces conditions. En toute rigueur, plus on s'éloigne d'elles, moins le modèle est valable. Contrairement donc à nos espoirs (DeCOURT, 1965) on ne peut construire de tables de production à éclaircies variables, définies arbitrairement, à l'aide du seul modèle proposé. La logique du modèle veut en effet qu'on reste proche des conditions moyennes de décroissance du nombre de tige. C'est heureusement ce qu'on a fait et ce qui permet de considérer le premier jeu de tables comme valable.

Par contre, en vertu même des hypothèses proposées, on peut, à partir de tables « moyennes », déjà construites, établir des tables correspondant à des éclaircies différentes, à l'aide de la méthode proposée par N. E. Hiley (Delvaux J., 1961) ou de méthodes voisines. La seule condition est alors d'être assuré que le régime d'éclaircie proposée n'entraîne pas de diminution des accroissements en volume total.

\section{3. - Possibilité d'évaluation directe de la production totale.}

Le raisonnement développé au $\S 1$, montre qu'on peut exprimer non seulement G et $\mathrm{G}_{\text {тот }}$, mais également $\mathrm{V}$ et $\mathrm{V}_{\text {тот }}$ en fonction de $\mathrm{H}$, de $\mathrm{N}$ et de $\mathrm{N}_{\mathrm{O}}$.

On aura : $\mathrm{V}_{\text {Tот }}=g\left(\mathbf{H}, \mathrm{N}_{\mathrm{O}}\right)$

$$
\mathrm{V}_{\mathrm{E}}=h\left(\mathrm{~N}-\mathrm{N}_{\mathrm{O}}, \mathrm{H}\right),(\mathrm{VE}=\text { Somme des volumes enlevés en éclaircie }) \text {. }
$$

et donc, pour le volume sur pied :

$$
\mathrm{V}=g\left(\mathrm{H}, \mathrm{N}_{\mathrm{o}}\right)-h\left(\mathrm{~N}-\mathrm{N}_{\mathrm{o}}, \mathrm{H}\right)
$$

c'est-à-dire pour un ensemble de peuplements où $\mathrm{N}_{0}$ est sensiblement constant

$$
\mathrm{V}=f(\mathrm{H}, \mathrm{N})
$$


L'ajustement direct, à partir des mesures faites dans les peuplements sur pied, de cette dernière relation permet d'évaluer $\mathrm{V}_{\text {тот }}$, Implicitement, $f$ contient en effet $g$ et $h$. Or si $\mathrm{N}=\mathrm{N}_{0}$, par construction $h=\mathrm{O}$ et on a

$$
\mathrm{V}_{\text {тот }}=g\left(\mathrm{H}, \mathrm{N}_{\mathrm{o}}\right)=f\left(\mathrm{H}, \mathrm{N}_{\mathrm{o}}\right)
$$

En théorie, la relation de Eichhorn s'obtient donc en remplaçant $\mathrm{N}$ par $\mathrm{N}_{\mathrm{O}}$ dans l'équation $f$.

Cependant, on ne perdra pas de vue que d'une part la relation $f$ est plus ou moins étroite et surtout que l'utilisation proposée ici est une extrapolation. En effet, dans les placettes mesurées $\mathrm{N}$ n'est jamais égal à $\mathrm{N}_{0}$, du fait soit des éclaircies, soit de la mortalité.

La forme de la relation $\mathrm{V}=f(\mathrm{H}, \mathrm{N})$ importe donc beaucoup, si on veut qu'elle reste valable lorsqu'on écrit $\mathrm{N}=\mathrm{N}_{0}$. Il serait logique, si la théorie développée ici est valable de lui donner une forme faisant explicitement apparaître un terme exprimant l'allure de la croissance de $\mathrm{V}_{\text {тот }}$ en fonction de $\mathrm{H}$ et un terme exprimant $\mathrm{V} \mathbf{E}$ et tendant vers zéro lorsque $\mathrm{N}$ tend vers $\mathrm{N}_{0}$. Des recherches devraient préciser la forme la plus adéquate de cette équation.

Appliquant la méthode à un ensemble de mesures instantanées, faites dans le dispositif permanent de la Hêtraie de Haye, dispositif comportant des placettes soumises à des éclaircies fortes, faibles ou nulles (témoin), on retrouve de façon très valable la relation production totale hauteur qu'on connaissait par ailleurs et cela pour des formes explicites diverses de l'équation $f$.

\section{4. - Conséquence des changements de densité de plantation dans l'utilisation des tables déjà construites}

Les tables existantes correspondent à une densité initiale moyenne des peuplements plus forte que celle pratiquée actuellement. L'analyse faite ci-dessus montre qu'elle risquent de s'appliquer mal à ces peuplements, d'origine plus récente. En fait, les équations-tarifs risquent d'être peu modifiées. De plus, la production totale (cf. discussion de l'hypothèse 1) est de moins en moins dépendante de $\mathrm{N}_{0}$ lorsque le peuplement se développe. On risque donc de constater des écarts surtout dans les jeunes peuplements, et pour la dimension des produits. Il faudra donc dans l'avenir réviser les tables, et sans doute modifier la méthode pour tenir compte notamment des différences de densité à l'origine. De toute façon, notre méthode n'est que provisoire et reste bien sûr très grossière. Pour construire des tables de production à régime d'éclaircie variable suffisamment précises, il faudra non seulement tenir compte des densités initiales, mais aussi préciser l'influence de la densité du peuplement sur son accroissement courant, comme essaient de le faire par exemple PiennaAr L. V. et Turnbull K. J. (1973), à partir du modèle de croissance de Von BertalanfFy.

Reçu pour publication en décembre 1973

\section{SUMMARY}

NOTE ON AN UNEXPECTED RELATIONSHIP IN FOREST MENSURATION. IMPORTANCE FOR THE METHOD USED TO CONSTRUCT THE YIELD-TABLES

The relationship, computed in pure even-aged stands, of different regions, refer to mean diameter, mean height and number of stems of the stand. She is a consequence of the nature of the 
stands and of the sylvicultural treatments. For a good use of this relation to construct the yieldtables, it is necessary to take into account these conditions to choose the plots and also the model used to compute the tables.

\section{ZUSAMMENFASSUNG}

\section{EINE UNERWARTETE HOLZMESSKUNDLICHE BEZIEHUNG UND IHRE METHODOLOGISCHE}

BEDEUTUNG FÜR DIE ERSTELLUNG VON ERTRAGSTAFELN.

Die untersuchte Beziehung, welche für gleichaltrige Reinbestände einer Holzart innerhalb eines Wuchsgebietes gilt, betrifft den Zusammenhang zwischen dem mittleren Umfang des Kreisflächenmittelstammes $(\mathrm{Cg})$, der mittleren Höhe des Kreisflächenmittelstammes $(\mathrm{Hg})$ und der Stammzahl. Diese Beziehung hängt von den untersuchten Beständen und deren waldbaulichen Behandlungen ab. Um sie bei der Erstellung von Ertragstafeln verwenden zu können, muss man diesen Bedingungen bei der Auswahl der Versuchsflächen und des Tafelmodeles Rechnung tragen.

\section{RÉFÉRENCES BIBLIOGRAPHIQUES}

Abetz, 1973. - Communication orale.

Anderson, J. O., 1963. - Produktion tabeller för norrländska tallplanteringar. Meddelanden fran statens skogsforskinstitut, Band 51, $\mathrm{n}^{\circ} 3$.

Assmann, 1961. - Waldertragskunde. BLV, München.

Bouchon J., 1973. Les tarifs de cubage. Doc. 73-3, Station Sylviculture et Production, C.N.R.F. Champenoux (54370) Einville.

Decourt, N., 1965. - Le Pin Sylvestre et le Pin Laricio de Corse en Sologne. Tables de production provisoires et méthodes utilisées pour les construire. (Ann. sci. Forest., 1965, t. XXII, Fas. 2). Voir aussi : Tables de production pour le Douglas (Ann. Sci. Forest. 1967), pour le Pin Maritime (Ann. sci. Forest., 1969), l'Epicéa Commun (Ann. Sci. Forest., 1972).

Decourt N., 1971. - Comparaison des équations de régression. Application au cubage des peuplements d'Épicéa Commun. Ann. Sci. Forest., 1971, 28 (1).

Decourt N., 1973. - Production primaire, production totale. Méthode d'évaluation. Indices de productivité. Ann. sci. Forest., 1973, 30 (4).

Decourt N., 1972. - Méthode utilisée pour la construction rapide de tables de production provisoires en France. Ann. Sci. Forest., 1972. 29 (1).

Delvaux J., 1961. - Calcul des éclaircies numériques (adopté et modifié d'après Hiley W. E.). Bulletin de la Société Royale Forestière de Belgique, 68e année, octobre 1961, 453-472.

EVERT F., 1971. - La prédiction de l'accroissement à partir de caractéristiques courantes du peuplement. Revue bimestrielle de Recherche du service des Forêts canadien, 1971, Vol. 27, no 5.

Kira T., Ogawa H., Sakazaki N., 1953. - Intraspecific competition among higher plants. I Competitionyield-density interrelationships in regularly dispersed population. Osaka City Univ. 4 (D). Inst. Polyt.

Kira T., Shinozaki, 1956. - Id. VII. Logistic theory of the C-D Effect. Inst. Polyt. Osaky City Univ. 7 (D).

Magin R., 1966. - Ertragsfestsetzung und Ertragsprognose in der Forsteinrichtung. 2. Internat. Ertragskunde tagung. Wien 1966. avec un calcul de F. Franz.

Piennaar L. V., Turnbull K. J., 1973. - The Chapman-Richards generalization of von BertalanfFy's growth model for basal area growth and yield in even-aged stands. Forest Science, 1973, vol. 19 (1). 